\title{
Recovery of bladder function in patients with acute spinal cord injury: significance of ASIA scores and somatosensory evoked potentials
}

Armin Curt, Biljana Rodic, Brigitte Schurch and Volker Dietz

Swiss Paraplegic Centre, University Hospital Balgrist, Zürich, Switzerland

\begin{abstract}
The significance of the ASIA (American Spinal Injury Association) scores and SSEP (somatosensory evoked potentials) recordings in predicting the recovery of bladder function was evaluated in 70 patients with acute, traumatic spinal cord injury (SCI). The patients were examined following admission to the rehabilitation centre (mean 10 days post-trauma) both clinically by the ASIA scores and electrophysiologically by tibial and pudendal SSEP recordings. The results of the initial examinations were related to the degree of recovery of bladder function of the patients assessed by urodynamic examination at the end of the rehabilitation programme (at least 6 months post-trauma). The recovery of somatic nerve function (external urethral sphincter function) involved in bladder function was correlated to both the initial ASIA scores and SSEP recordings (Spearman correlation, $P<0.001$ ). The latter parameters, however, were not related to the outcome of autonomic nerve function (eg detrusor vesicae function) (Spearman correlation, $P=0.1$ ). Therefore, the initial clinical and electrophysiological examinations are of value in assessment of the degree to which the patient will recover somatic nervous control of bladder function. However, these examinations are not indicative of urodynamic impairment. Therefore, urodynamic examination should be mandatory for the diagnostic assessment and therapeutical approach of bladder dysfunction in patients with acute SCI.
\end{abstract}

Keywords: spinal cord injury; ASIA protocol; SSEP; urinary bladder function

\section{Introduction}

In patients with traumatic spinal cord injury (SCI) clinical examination is the first and most important approach in the diagnostic assessment of the patient. The ASIA protocol (inaugurated by the American Spinal Injury Association), which is based on longlasting clinical experience, allows the clinician to perform a standardized clinical examination in order to assess the extent of SCI and to predict to some degree the prognosis of functional disability. ${ }^{1-3}$ Furthermore, in combination with SSEP recordings the diagnostic assessment can be improved, especially in incomprehensive and uncooperative patients. ${ }^{4-6}$ Therefore, the combination of ASIA scores and SSEP recordings can be of high value in predicting the outcome of ambulatory capactity ${ }^{7-9}$ or hand function $^{10}$ in acute SCI patients. In line with these observations, the aim of this study was to evaluate the degree to which initial ASIA scores and SSEP recordings are related to the recovery of bladder function in patients with acute SCI. However, in micturition and bladder function both the somatic and autonomic nervous systems are involved. Therefore, we related the results of the initial examinations

Correspondence: A Curt to the recovery of (1) bladder function according to the urodynamic examination and classification. This depends on autonomic and somatic nerve function and (2) the external utethral sphincter function (EUS), which depends on somatic nerve function. This approach should demonstrate the correlation of initial clinical and electrophysiological examinations with respect to the impairment of the somatic and autonomic nervous system involved in bladder function.

\section{Methods}

General procedures and patients

Patients with acute traumatic para- $(n=39 ; 9$ female, 30 male; mean age $40 \pm 16$ years) and tetraplegia ( $n=31 ; 4$ female, 27 male; mean age $43 \pm 22$ years) who were hospitalized for rehabilitation in the Paraplegic Centre between 1993-1995 were studied. Only patients with ASIA impairment Grade A-D were included (Tables 1 and 2). Patients with nontraumatic diseases of the peripheral nervous system (eg diabetes mellitus, peripheral nerve entrapments) or cerebral lesions (traumatic and non-traumatic) were excluded. Fractures of the spine were verified radio- 
Table 1 ASIA impairment Grades and mean ASIA scores (with standard deviation) from the group of paraplegic patients

\begin{tabular}{lcccrcr}
\hline ASIA & \multicolumn{2}{c}{ Motor scores } & \multicolumn{2}{c}{ Light touch scores } & \multicolumn{2}{c}{ Pin prick scores } \\
Impairment grade & mean & $S D$ & mean & SD & mean & SD \\
\hline A $(n=17)$ & 51.3 & 3.06 & 73.2 & 13.23 & 70.9 & 12.56 \\
B $(n=7)$ & 53.6 & 5.47 & 91.9 & 9.11 & 82.9 & 15.41 \\
C $(n=10)$ & 66.4 & 10.04 & 95.1 & 10.51 & 90.9 & 16.41 \\
D $(n=5)$ & 85.1 & 11.71 & 105.1 & 8.72 & 104.4 & 9.53 \\
\hline
\end{tabular}

Table 2

\begin{tabular}{lcccccc}
\hline ASIA & \multicolumn{2}{c}{ Motor scores } & \multicolumn{2}{c}{ Light touch scores } & \multicolumn{2}{c}{ Pin prick scores } \\
Impairment grades & mean & $S D$ & mean & SD & mean & SD \\
\hline A $(n=11)$ & 18.9 & 15.71 & 33.2 & 22.44 & 23.1 & 10.11 \\
B $(n=5)$ & 16.6 & 12.68 & 50.4 & 16.65 & 38.6 & 15.87 \\
C $(n=7)$ & 44.1 & 25.19 & 93.6 & 19.97 & 86.2 & 30.32 \\
D $(n=8)$ & 79.9 & 9.66 & 99.2 & 15.98 & 87.1 & 32.97 \\
\hline
\end{tabular}

Same parameters as shown in Table 1 from the group of tetraplegic patients

logically, eg by X-ray, computer tomography (CT) and/or magnet resonance imaging (MRI). Patients with a neurological level of lesion $\mathrm{C} 4-\mathrm{T} 1$ were classified as tetraplegic whereas those with a level below T1 were classified as paraplegic.

\section{ASIA score}

A full neurological examination of all patients was performed according to the ASIA protocol (marked as 'full ASIA score'). In patients with acute SCI two examinations were performed. The first was initially after hospitalization in the Paraplegic Centre and the second 6 months post-trauma. In order to evaluate more specifically the significance of the ASIA protocol in the prediction of bladder recovery a so-called 'sacral ASIA score' was recorded. This was related to sensory impairment in dermatomes $\mathrm{S} 2-\mathrm{S} 5$ according the ASIA protocol (pin prick and light touch; maximum of points 12) and motor functions of the legs (maximum of points 50). The latter scores were evaluated in parallel with the 'full ASIA score'.

\section{Assessment of bladder recovery}

The recovery of bladder function of the patients was assessed by urodynamic examination. As this study was focussed on the level of recovered bladder function, urodynamic examinations were performed in the chronic stage of SCI, at least 6 months post-trauma or at the end of the rehabilitation programme. The degree of recovery of bladder function was assessed and categorized in two ways. On one hand by urodynamic classification, which corresponds to those used in earlier studies ${ }^{11,12}$ and focusses on urodynamic and neurogenic characteristics of bladder dysfunction, on the other hand by the assessment of external urethral sphincter function (EUS). EUS function was graded into three categories: (1) Normal. This category is characterized by normal voluntary EUS, sensory and detrusor function; (2) Impaired function. This is characterized by preserved voluntary EUS function, but disturbed micturition due to impaired sensoryand/or detrusor function; (3) Absence of function. This is characterized by a loss of voluntary EUS function and impaired sensory and detrusor function. This gradation demonstrates the preservation of voluntary EUS function, which is a major aspect of physiological micturition. Furthermore, voluntary EUS function depends on somatic nerve function, while the bladder function and urodynamic classification depend on the function of both the somatic and autonomic nervous systems.

\section{Urodynamic examination}

Measurements of the post-void volume and cultures of the urine were obtained before each urodynamic examination. Videocystourethromanometry was performed while the patients were in supine position with the pelvis tilted 15 degrees. Two microtransducer catheters (No 5 and 3 radioopaque, 8F) were used for the urethrovesical and anorectal pressure recordings, respectively. ${ }^{13}$ Proper localization of the transducers within the bladder, bladder neck, and urethra in relation to the anatomical landmarks of the pelvis were confirmed using an image intensifier. Slow bladder filling $(5 \mathrm{ml} / \mathrm{min})$ was used with a $24 \%$ contrast medium (3-acetylamino-methyl-5-acetylamino-2,4,6-tribenzoic acid). Drugs that could interfere with the urethrovesical functions were discontinued $24 \mathrm{~h}$ before examination. Urodynamic examinations were performed according to the recommendations of the Urodynamic Society. ${ }^{14}$ Neurogenic bladder dysfunction due to upper and lower motoneuron lesions were defined according to the recommendations of the 
International Continence Society. ${ }^{12}$ In some difficult cases the ice water test, urecholine test or Susset test was performed during the videocystomanometry in order to confirm diagnosis. ${ }^{15-17}$

\section{SSEP recordings}

The electrophysiological recordings were performed in parallel with the clinical examinations. Tibial somatosensory evoked potentials (SSEP) were elicited by electrical stimulation $(0.2 \mathrm{msec}$ duration square wave applied at $3 \mathrm{~Hz}$ ) of the tibial nerves of both legs, delivered at the medial ankle with the cathode placed $2-3 \mathrm{~cm}$ proximal to the anode. Pudendal SSEP were elicited by the same stimulation parameters using ring electrodes attached to the penis in men and with skin electrodes at the level of clitoris in women as described elsewhere. ${ }^{18,19}$ Subjects were lying prone at ambient room temperature. The stimulus intensity was adjusted (up to $40 \mathrm{~mA}$ ) to produce a clear muscle contraction or sensation. The recording electrodes (conventional $0.5 \mathrm{~cm}$ gold cup electrodes) were attached to the skin over the popliteal fossa in tibial SSEP (in order to control the effectiveness of electrical stimulation and transmission of the potentials along the peripheral nerve segments). Scalp electrodes were positioned at $\mathrm{Cz}^{\prime}-\mathrm{Fz}$ (international $10 / 20$ electrode system). The electrode impedance was maintained at less than $5 \mathrm{k} \Omega$. The amplifier was set at $5 \mu \mathrm{V} /$ div and the time of analysis at $60 \mathrm{~ms}$. Two sets of 500 responses were averaged and superimposed to ensure consistency.

The P40 latency and amplitude (base to peak) of the SSEP recordings were determined and the results were classified into 5 categories: ${ }^{10,20}$ Category 1 ; no cortical SSEP recording. Category 2; pathologic P40 latency and amplitude. Category 3; delayed P40 latency with normal amplitude. Category 4; normal P40 latency with reduced amplitude. Category 5; normal P40 latency and amplitude. Such a procedure reflects the different degrees of impaired impulse transmission within the spinal cord.

Control data were collected from 10 healthy subjects (five male, five female, mean age 29.6 years, range $24-38$ years). Because the P40 latency is related to the body height, the limits to define significant pathological results of the P40 latency were related to the control data corrected for height (latency $=0.199 \times$ (body height-6.97)). The limits for the P40 amplitude (base to peak) was set to $>0.5 \mathrm{mV}$. These limits are in accordance with values reported elsewhere. ${ }^{21}$

\section{Statistics}

Statistical evaluations were performed using a SAS (Information Delivery System) software package. Spearman correlation analysis (level of significance, $P<0.001$ ) and Student $t$-test (level of significance, $P<0.05)$ were performed to evaluate statistical significance.

\section{Results}

\section{Acute SCI patients}

In 31 tetraplegic patients and 39 paraplegic patients the first clinical (ASIA scores) and electrophysiological (SSEP) examinations were performed within an average of 10 days (range $1-35$ days) post-trauma and was repeated after 6 months. Therefore, the present study is concerned with the early phase of rehabilitation and has to be distinguished from studies referring to examinations immediately post trauma. The results of the initial clinical and electrophysiological examinations were related to the recovery of bladder function (urodynamic classification) and voluntary EUS function of these patients after discharge from the rehabilitation programme (at least 6 months post-trauma).

\section{Recovery of bladder function}

In patients with acute tetraplegia $27 \%$ recovered to normal bladder function according to the urodynamic classification, $73 \%$ developed an incomplete $(40 \%)$ or complete $(33 \%)$ neurogenic bladder dysfunction characteristic of upper motor neuron lesion (UMN). There was no patient with a secondary lesion of the conus medullaris. The voluntary EUS function was assessed to be normal in $27 \%$, impaired in $39 \%$ and absent in $34 \%$ of patients.

In the paraplegic patients only $10 \%$ recovered to normal bladder function (urodynamic classification), $47 \%$ developed a bladder dysfunction characteristic of upper motor neuron lesion (UMN) $(40 \%$ complete, $7 \%$ incomplete), 33\% characteristic of lower motor neuron lesion (LMN) (13\% complete, $20 \%$ incomplete) and $8 \%$ a combination of UMN and LMN. The voluntary EUS function was assessed to be normal in $10 \%$, impaired in $30 \%$ and absent in $60 \%$ of patients.

\section{Clinical examination and recovery of bladder function} In patients with acute tetraplegia both the initial 'full ASIA score' and the 'sacral ASIA score' were significantly correlated (Spearman correlation analysis; $P<0.001)$ to the recovery of both bladder function (urodynamic classification) and voluntary EUS function (Table 3). There was no significant difference between the predictive value of the 'full ASIA score' (Spearman corr. coeff. $=0.57 ; P<0.001$ ) and the 'sacral ASIA score' (Spearman corr. coeff. $=0.58$; $P<0.001)$. The statistical analysis revealed no significant difference between pin prick and light touch (pin prick: corr. coeff. $=0.51, P<0.001$; light touch: corr. coeff. $=0.60, \quad P<0.001)$. The tetraplegic group consisted only of patients with neurogenic bladder dysfunction due to UMN lesion. Only $10 \%$ of the patients with loss of pin prick and light touch of the sacral dermatomes at the initial clinical examination retained incomplete bladder dysfunction, and none of these patients recovered normal bladder function. All 
patients who finally achieved normal bladder function had an initial 'sacral ASIA score' of at least six for light touch and pin prick.

In the acute paraplegic patients the initial full and sacral ASIA scores were significantly correlated to the recovery of voluntary EUS function (student $t$-test, $P<0.001$ ), but not to the recovery of bladder function according to the urodynamic classification (student $t$ test; $P=0.1$ ). Although the ASIA scores predicted the recovery of voluntary EUS function, both the full and sacral ASIA scores did not differentiate between neurogenic bladder dysfunction from the type of UMN or LMN lesion (urodynamic classification) (Table 4). In the paraplegic group, patients with complete loss of voluntary EUS function had a loss of sacral pin prick at the initial clinical examination in $90 \%$ of cases, while only $75 \%$ presented a complete loss of sacral light touch sensation. Due to the relatively small group of ASIA B patients, this difference was not statistically significant. Similar to the tetraplegic patients, only $10 \%$ of the patients with initial complete loss of sacral pin prick and light touch finally developed an incomplete bladder dysfunction at 6 months post-trauma.

SSEP recordings and recovery of bladder function In the tetraplegic patients both the initial tibial SSEP recordings and the clinical examination were significantly related to the outcome of bladder function (with respect to both the urodynamic classification and voluntary EUS function; Spearman corr. coeff. 0.50, $P<0.003$ ) (Figure 1).

The initial pudendal SSEP recordings in the tetraplegic patients were not significantly related to the outcome of bladder function (Spearman correlation, $P=0.1)$, as in $20 \%$ of the patients pudendal SSEP could not be recorded although the patients did not show a complete sacral deafferentation (false negative results). However, in contrast to the tibial SSEP all of the patients in whom initially a pudendal SSEP could be recorded, recovered to at least an incomplete bladder dysfunction.

In the paraplegic patient group both the initial tibial (Spearman correlation, $P<0.007$ ) and pudendal (Spearman correlation, $P<0.0007)$ SSEP recordings were significantly related only to the outcome of EUS function (Figure 2). Similar to the clinical examination, the SSEP recordings predicted the recovery of voluntary EUS function, but were not related to the urodynamic dysfunction (differentiation between UMN or LMN lesion) (Spearman correlation, $P=0.1$ ). Of the paraplegic group $90 \%$ of patients with an absence of pudendal SSEP at the initial

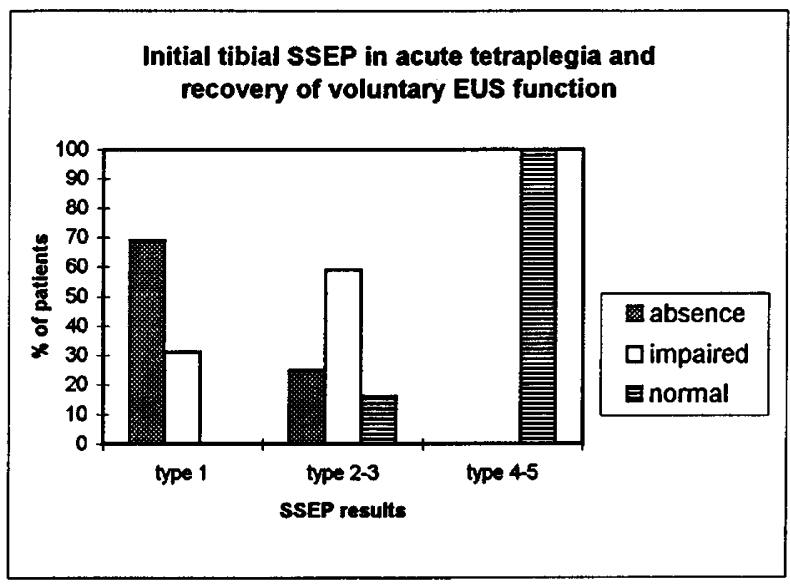

Figure 1 Initial tibial SSEP recordings in acute tetraplegia and recovery of voluntary EUS function (SSEP result: type 1 $=$ no SSEP; type $2-3=$ pathological latency; type $4-5$ normal latency)

Table 3 Relation between the initial 'sacral ASIA score' (mean and standard deviation) in patients with acute tetraplegia and recovery of voluntary EUS function

\begin{tabular}{llccr}
\hline Initial 'sacral ASIA score' & & Absence & $\begin{array}{c}\text { Recovery of voluntary EUS function } \\
\text { Impaired }\end{array}$ & Normal \\
\hline Sacral light touch S2 - S5 & $(\max 12$ points) & $1 \pm 2$ & $6 \pm 5$ & $10 \pm 3$ \\
Sacral pin prick S2 - S5 & $(\max 12$ points) & 0 & $5 \pm 4$ & $9 \pm 3$ \\
Motor score of the legs & $(\max 50$ points) & 0 & $29 \pm 23$ & $46 \pm 6$ \\
\hline
\end{tabular}

Table 4 In acute paraplegia the recovery of voluntary EUS function was correlated with the 'sacral ASIA score' (mean and standard deviation), but there was no relation to the kind of urodynamic dysfunction (upper vs lower motor neuron lesion)

\begin{tabular}{|c|c|c|c|c|c|}
\hline \multirow{2}{*}{$\begin{array}{l}\text { Initial 'sacral ASIA score' and: } \\
\text { recovery of EUS function } \\
\text { Recovery of bladder dysfunction }\end{array}$} & & \multicolumn{2}{|c|}{ Absence } & \multicolumn{2}{|c|}{ Impaired } \\
\hline & & $U M N$ & $L M N$ & $U M N$ & $L M N$ \\
\hline Sacral light touch & $(\max 12)$ & $2.5 \pm 3.3$ & $1 \pm 1.2$ & $7.3 \pm 1.9$ & $7.3 \pm 3.9$ \\
\hline Sacral pin prick & $(\max 12)$ & $0.8 \pm 2$ & $0 \pm 0$ & $5.3 \pm 4.1$ & $7.2 \pm 3.8$ \\
\hline Motor score of the legs & $(\max 50)$ & $3.5 \pm 6.9$ & $3.4 \pm 4.1$ & $7.3 \pm 5.2$ & $25 \pm 15.8$ \\
\hline
\end{tabular}




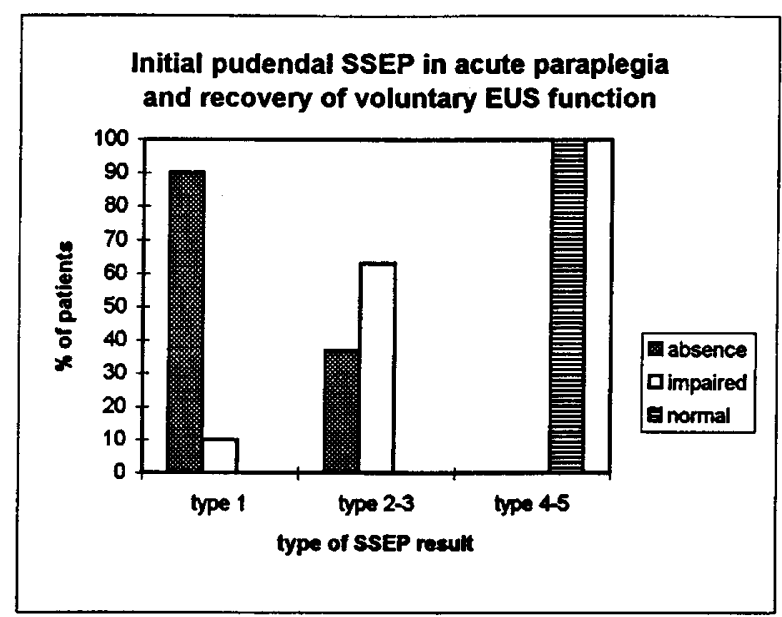

Figure 2 Initial pudendal SSEP in acute paraplegia and recovery of voluntary EUS function (SSEP result: type $1=$ no SSEP; type 2-3=pathological latency; type $4-5=$ normal latency)

examination finally retained a complete loss of voluntary EUS function, while all patients with preserved pudendal SSEP recordings recovered to normal or at least an impaired voluntary EUS function. The pudendal SSEP were more sensitive than the tibial SSEP recordings to predict EUS function, as $33 \%$ of patients with normal tibial SSEP nevertheless developed an impaired EUS function. In contrast to the tetraplegic patients, no significant percentage of the paraplegic patients showed false negative pudendal SSEP recordings in patients with incomplete SCI lesion.

\section{Discussion}

Impaired bladder function is one of the most relevant complications in patients with complete and incomplete SCI. The introduction of new diagnostic and therapeutic techniques allows the optimisation of the treatment of neurogenic bladder dysfunction and improves rehabilitation and life expectancy of SCI patients. ${ }^{22,23}$ Therefore, it is important to assess the type of bladder dysfunction at an early stage in patients with acute SCI. Cystometric examinations in combination with radiography are the most sensitive techniques used to assess those parameters reflecting urodynamic (detrusor and sphincter pressure etc.) and morphologic characteristics of a normal or impaired bladder function. ${ }^{24,25}$ This allows an appropriate diagnosis and management of voiding dysfunction in patients with neurogenic bladder dysfunction. ${ }^{11,26}$

The clinical examination according to the ASIA protocol are restricted to the assessment of somatic motor and sensory nerve functions in patients with acute SCI. Therefore, this examination can only be of partial value in patients with neurogenic bladder dysfunction, as the autonomic part of bladder dysfunction is not assessed. Nevertheless, the clinical examination (ASIA scores and Impairment Grade) is usually the first diagnostic approach in acute SCI and therefore, it is of interest to know its value in the prediction of bladder function. ${ }^{1,2,27}$

The diagnosis of bladder (voiding) and sexual (impotence) dysfunction in neurological disorders (multiple sclerosis) with electrophysiological techniques is of restricted value, as the autonomic nervous system is not evaluated by electromyography (EMG, reflexes) or somatosensory evoked potentials (tibial and pudendal SSEP). ${ }^{28-31}$ Therefore, in the latter patients these electrophysiological recordings show the best correlation to disturbances of somatic nerve functions. ${ }^{32}$

This was evident in the present study. It was demonstrated, that the impairment of bladder function due to somatic nerve dysfunction (voluntary EUS function) could be predicted by clinical and electrophysiological means in both paraplegic and tetraplegic patients. Therefore, the latter examinations are similarly related with the recovery of EUS function as with the outcome of other somatic nerve functions, eg ambulatory capacity and hand function..$^{7-10}$

These results are, however, of limited value in predicting the recovery of urodynamic bladder function, because both the full and sacral ASIA scores and the SSEP recordings cannot indicate the extent to which the autonomic nervous system is disturbed and whether bladder dysfunction is due to an upper or lower motor neuron lesion (detrusor function). The correlation of the clinical and electrophysiological examinations with the urodynamic bladder function in the tetraplegic patient group is due to the fact, that in the latter group there were no patients with secondary lesions of the conus medullaris. It is evident from the results of the paraplegic patient group, that the latter examinations do not allow to assess the autonomic nerve function involved in bladder function.

Additional recordings of lumbar SSEP can indicate lesions of the conus medullaris, which are related to detrusor dysfunction. However, these recordings also failed to give information about the urodynamic characteristics (hyper- $v s$ hypo-reflexive detrusor). ${ }^{33,34}$ The best informations can be obtained by the combination of both urodynamic and electrophysiological examinations. ${ }^{35-41}$

\section{Conclusions}

Although there is a limited relationship between the results of the initial clinical and electrophysiological examinations and the recovery of urodynamic bladder function, they are of clinical relevance. ${ }^{41}$ They assess the impairment of bladder function due to the voluntary EUS function in acute SCI patients and can indicate already early after trauma the degree to which EUS function is likely to recover. This can be of additional value in patients with acute SCI, where the urodynamic characteristics are altered due to spinal shock. $^{42}$ 


\section{Acknowledgements}

We thank Dr I Gibson for editorial service and Mr T Erni for statistical support. This work was supported by the Swiss National Science Foundation (Grant No. 3142899.95) and the Int. Research Institute for Paraplegia (P16/93).

\section{References}

1 Ditunno JF, Young W, Donovan WH, Creasey G. The international standards booklet for neurological and functional classification of spinal cord injury. Paraplegia 1994; 32: $70-80$.

2 Donovan WH et al. A Test of the ASIA Guidelines for Classification of Spinal Cord Injuries. J Rehab 1990; 4: 39-53.

3 Bednarczyk JH, Sanderson DJ. Comparison of functional and medical assessment in the classification of persons with spinal cord injury. J Rehab Res 1993; 30: 405-411.

4 Grundy BL, Friedman W. Electrophysiological Evaluation of the Patient with Acute Spinal Cord Injury. Crit Care Clin 1987; 3: $519-548$.

5 Houlden DA, Schwart ML, Klettke KA. Neurophysiologic diagnosis in uncooperative trauma patients: confounding factors. J Trauma 1992; 33: 244-251.

6 Kamimura $\mathrm{N}$ et al. Spinal somatosensory evoked potentials in infants and children with spinal cord lesions. Brain Dev 1988; 10: $355-359$.

7 Jacobs SR, Yeaney NK, Herbison GJ, Ditunno JF. Future ambulation prognosis as predicted by somatosensory evoked potentials in motor complete and incomplete Quadriplegia. Arch Phys Med Rehabil 1995; 76: 635-641.

8 Crozier KS et al. Spinal cord injury: prognosis for ambulation based on quadriceps recovery. Paraplegia 1992; 30: $762-767$.

9 Curt A, Dietz V. Traumatic cervical spinal cord injury: relation between somatosensory evoked potentials, neurological deficit and hand function. Arch Phys Med Rehabil 1996; 77: $48-53$.

10 Curt A, Dietz V. Ambulatory capacity in spinal cord injury: significance of somatosensory evoked potentials and ASIA protocols in predicting outcome. Arch Phys Med Rehabil 1997; 78: $39-43$.

11 Bors E, Comarr AE. Neurological Urology. University Park Press, Baltimore 1971, pp 129-135.

12 Bates CP et al. The standardization of terminology of lower urinary tract function. J Urol 1979; 12: $551-557$.

13 Rossier AB, Fam BA. 5-microtransducer catheter in evaluation of neurogenic bladder function. Urology 1986; 17: $371-$ 378 .

14 Blaivas JG et al. Urodynamic procedure recommendations of the Urodynamic Society. I. Procedures that should be available for routine practise. Neurourol Urodyn 1982; 1: 51-55.

15 Bors E. Neurogenic bladder. Urol Survey 1957; 7: 177-250.

16 Lapides J, Friend CR, Ajemian EP, Reus WS. Denervation supersensitivity as a test for neurogenic bladder. Surg Gyn Obst 1962; 114: $241-244$.

17 Susset JG, Ghoniem GM, Regnier CF. Rapid cystometry in males. Neurourol Urodyn 1982; 1: 319-327.

18 Haldeman S, Bradley WE, Bhatia NN, Johnson BK. Pudendal evoked responses. Arch Neurol 1982; 39: 280-283.

19 Opsomer RJ et al. Pudendal cortical somatosensory evoked potentials. J Urol 1986; 135: 1216-1218.

20 Kovindha A, Mahachai R. Short-latency somatosensory evoked potentials (SSEP) of tibial nerves in spinal cord injuries. Paraplegia 1992; 30: 502 - 506.
21 Desmedt JE, Noel P. Average cerebral evoked potentials in the evaluation of lesions of the sensory nerves and of the central somatosensory pathway. In: Desmedt JE (ed). New Developments in Electromyography and Clinical Neurophysiology, vol 2. Basel: Karger, 1973, pp 352-371.

22 Yalla SV, Fam AB. Spinal cord injury. In: Krane RJ and Siroky MB (eds). Clinical Neurourology 2nd ed. Boston: Little, Brown and Co, 1991, pp 319-332.

23 Schurch B et al. Botulinum-A toxin as a teratment of detrusorsphincter dyssynergia: a prospective study in 24 spinal cord injury patients. J Urol 1996; 155: 1023-1029.

24 Gibbon NOK. Nomenclature of neurogenic bladder. Urology 1976; 8: 423 - 429.

25 Krane RJ, Siroky MB. Classification of neurogenic-urologic disorders. In: Clinical Neuro-Urology. Little, Brown \& Co. Boston 1979, pp $143-158$

26 Lapides J. Neuromuscular vesical and urethral dysfunction. In: Campbell, MF, Harrision JH (eds). Urology 3 ed, vol 2. Saunders, Philadelphia 1970, pp 1343-1379.

27 Weiss DJ et al. Correlation of bladder function after spinal cord injury with initial perianal pinprick testing. J AM Paraplegia Soc 1994; 17(2): 106.

28 Pickard RS, Powell PH, Schofield IS. The clinical application of dorsal penile nerve cerebral-evoked response recording in the investigation of impotence. Br J Urology 1994; 74: 231-235.

29 Nogueira MC, Herbaut AG, Wespes E. Neurophysiological investigations of two hundred men with erectile dysfunction. Eur Urol 1990; 18: $37-41$.

30 Kunesch E et al. Neurological risk profile in organic erectile impotence. J Neurol Neurosurg Psychiat 1992; 55: 275-281.

31 Betts CD, Jones SJ, Fowler CG, Fowler CJ. Erectile dysfunction in multiple sclerosis: Associated neurological and neurophysiological deficites, and treatment of the condition. Brain 1994; 117: $1303-1310$.

32 Deldovici ML, Fowler CJ. Clinical value of the pudendal somatosensory evoked potential. Electroenceph Clin Neurophys 1995; 96; $509-515$.

33 Beric A, Light JK. Correlation of bladder dysfunction and lumbosacral somatosensory evoked potential s-wave abnormality in spinal-cord-injured patients. Neurourol Urodynamics 1988; 7: $131-140$.

34 Zanette et al. Neurophysiological and urodynamic examinations in the functional assessment of spinal cord below the injury site. Eur Neurol 1995; 35: $93-98$.

35 Fowler CJ. Clinical significance of electrophysiological studies of patients with lower urinary tract dysfunction. Neurourol Urodynamics 1992; 11: 279-282.

36 Blaivas JG, Sinha HP, Zayed AAH, Labib KB. Detrusorexternal sphincter dyssynergia: a detailed electromyographic study. J Urol 1981; 125: 545-548.

37 Venetz U, Casanova G, Hess CW, Ludin HP. Kombinierte urodynamisch-elektromyographische Untersuchung bei Patienten mit multiper Sklerose und Blasenstörungen. Nervenarzt 1989; 60: $163-167$

38 Lucas MG, Thomas DG. Lack of relationship of conus reflexes to bladder function after spinal cord injury. BrJ Urol 1989; 63:24-27.

39 Susset JG, Ghoniem GM. Rapid cystometry and sacral-evoked response in the diagnosis of peripheral bladder and sphincter denervation. J Urol 1984; 132: 704-707.

40 Swash M, Snooks SJ. Slowed motor condution in lubosacral nerve roots in cauda equina lesions: a new diagnostic technique. $J$ Neurol Neurosurg Psychiat 1986; 49: 808 -816.

41 Gleave JR, Macfarlane R. Prognosis for recovery of bladder function following lumbar central disc prolapse. Br J Neurosurg 1990; 4: 205-210.

42 Rossier AB, Ott R. Bladder and urethral recordings in acute and chronic spinal cord injury patients. Urol Int 1976; 31: $49-$ 59 . 